\title{
Genetic and Environmental Risk Factors for Age-Related Macular Degeneration in Persons 90 Years and Older
}

\author{
Lebriz Ersoy, ${ }^{1}$ Tina Ristau, ${ }^{1}$ Moritz Hahn, ${ }^{2}$ Marcus Karlstetter, ${ }^{1}$ Thomas Langmann, ${ }^{1}$ \\ Katharina Dröge, ${ }^{1}$ Albert Caramoy, ${ }^{1}$ Anneke I. den Hollander, ${ }^{3}$ and Sascha Fauser ${ }^{1}$ \\ ${ }^{1}$ Department of Ophthalmology, University Hospital of Cologne, Cologne, Germany \\ ${ }^{2}$ Institute of Medical Statistics, Informatics and Epidemiology, University of Cologne, Cologne, Germany \\ ${ }^{3}$ Departments of Ophthalmology and Human Genetics, Radboud University Nijmegen Medical Centre, Nijmegen, The Netherlands
}

Correspondence: Sascha Fauser, University Hospital of Cologne, Department of Ophthalmology, Kerpener Strasse 62, 50924 Cologne, Germany;

Sascha.fauser@uk-koeln.de.

Submitted: October 11, 2013

Accepted: February 17, 2014

Citation: Ersoy L, Ristau T, Hahn M, et al. Genetic and environmental risk factors for age-related macular degeneration in persons 90 years and older. Invest Ophthalmol Vis Sci. 2014;55:1842-1847. DOI:10.1167/ iovs.13-13420
Purpose. We studied associations of genetic polymorphisms in age-related maculopathy susceptibility 2 (ARMS2) and complement factor $\mathrm{H}(C F H)$ in nonagenarians with age-related macular degeneration (AMD).

Methods. This case-control study comprised 2737 persons (1204 controls, 1433 AMD cases), including 166 nonagenarians (52 controls, 114 AMD cases). Single nucleotide polymorphisms (SNPs) in the genes ARMS2 and $C F H$ were determined. Risk scores were computed by multiple logistic regression analysis, including genetic and environmental risk factors (smoking, hypertension, body mass index, diabetes) for different age groups $(<70,70-79$, $80-89, \geq 90$ years [nonagenarians]).

RESULTs. In nonagenarians, ARMS 2 showed the weakest associations with AMD (odds ratio $[\mathrm{OR}]=1.52, P=0.127)$ compared to the other groups $\left(\mathrm{OR}, 70\right.$ years $=2.23, P=1.03 \times 10^{-13}$; OR, 70-79 years $=2.70, P=1.00 \times 10^{-13}$; OR, 80-89 years $=3.11, P=6.56 \times 10^{-8}$ ). For $C F H$, ORs for AMD increased with age $\left(<70\right.$ years OR $=1.96, P=1.80 \times 10^{-11} ; 70-79$ years OR $=1.89, P=4.48 \times 10^{-13} ; 80-89$ years $\left.\mathrm{OR}=2.71, P=1.28 \times 10^{-7}\right)$, but decreased again in the nonagenarians $(\mathrm{OR}=2.21, P=0.005)$. Compared to the group $<70$ years, reduced minor allele frequencies (MAFs) for AMD patients were observed in the nonagenarians ( $C F H 0.54$ vs. 0.43, $P$ $=0.009$; ARMS2 0.44 vs. $0.29, P=2.97 \times 10^{-5}$ ), while the MAFs in controls were not significantly different. The genetic risk score revealed the lowest discriminative power in the nonagenarians with an area-under-curve (AUC) of 0.658 for receiver-operating characteristics (AUC 80-89 years $=0.768,70-79$ years $=0.704,<70$ years $=0.682)$, while no significant difference was seen for the environmental risk score $($ AUC $<70$ years $=0.579,70-79$ years $=$ $0.567,80-89$ years $=0.600,>90$ years $=0.608$ )

Conclusions. Risk alleles in $C F H$ and ARMS2 have a significantly smaller effect on AMD development in nonagenarians, while environmental factors retain a similar effect.

Keywords: age-related macular degeneration, age, genetics

\begin{abstract}
A ge-related macular degeneration is one of the most A common age-related diseases and the leading cause of severe vision impairment in developed countries. Vision loss occurs mostly in advanced stages. either due to geographic atrophy of the retinal pigment epithelium or due to neovascular AMD with the formation of choroidal neovascularization (CNV). Although the etiology of AMD is known to be multifactorial, involving a complex interaction between genetic predisposition and environmental factors, such as age, cigarette smoking, body mass index (BMI), diabetes, and hypertension, ${ }^{1-8}$ the genetic variants associated with AMD account for approximate1y $70 \%$ of the risk for the condition. ${ }^{9,10}$ Hence, substantial effort has been made in understanding the genetics of AMD by identifying several AMD susceptibility loci over the past years. The two major loci were identified at chromosomes $1 \mathrm{q} 31$ and 10q26. These two loci explain approximately half of the heritability of AMD. ${ }^{11}$ They involve variants in the complement factor $\mathrm{H}(\mathrm{CFH})$ gene, the main regulator of the alternative complement pathway, ${ }^{12}$ and polymorphisms on chromosome 10q26 encompassing the age-related maculopathy susceptibil-
\end{abstract}

ity 2 (ARMS2) gene, ${ }^{13}$ and the adjacent high-temperature requirement factor A1 (HTRA1) gene, ${ }^{14}$ which may alter the integrity of Bruch's membrane ${ }^{15}$ Age is of high importance in the pathogenesis of AMD, with a prevalence of AMD in nonagenarians of almost $60 \%{ }^{16}$ Although genetic studies adjusted for age as a confounder, most studies had only low numbers of very old participants aged over 90 years.

In this study, the impact of genetic associations and environmental influences in nonagenarian AMD patients in comparison with other age groups was investigated. For this purpose, four different age groups $(<70,70-79$, and $80-89$ years, and nonagenarians) were analyzed for risk variants in CFH and ARMS2, and known environmental risk factors, such as hypertension, BMI, cigarette smoking, and diabetes mellitus.

\section{Patients and Methods}

\section{Study Population}

The current study was part of the European Genetic Database (EUGENDA, available in the public domain at www.eugenda. 
org), and included 166 nonagenarian persons (147 from Cologne area, 19 from Nijmegen area) and 2571 persons aged between 50 and 89 years $(984$ persons $<70$ years, 1099 persons aged $70-79$ years, 488 persons aged $80-89$ years; 1218 from Cologne, 1353 from Nijmegen). EUGENDA is a GermanDutch database for AMD patients and healthy control individuals, and comprises more than 4000 phenotyped cases. For the recruitment of nonagenarians, 1500 persons of 5314 aged 90 years and older from the registry of the city of Cologne were chosen by random and contacted once by mail. No information except their age was available for contacted persons and for the persons not participating in this study. ${ }^{16}$

All participants gave written consent before inclusion in the study. From all participants peripheral blood samples were collected, and detailed information about medical history, and dietary and life-style habits, such as smoking, were documented through a questionnaire. Retinal imaging was performed using color fundus photography (FP) of Field 2 (Cologne: Canon UVI fundus camera; Canon, Tokyo, Japan, and Nijmegen: Topcon TRC 50IX fundus camera; Topcon, Tokyo, Japan). Spectral-domain optical coherence tomography (SDOCT; Spectralis HRA system; Heidelberg Engineering, Heidelberg, Germany) and fluorescein angiography (FA) images were evaluated additionally if available.

Collection of data was performed in accordance with the tenets of the Declaration of Helsinki and the Medical Research Involving Human Subjects Act (WMO) and approved by the local ethics committees of the participating centers at Cologne and Nijmegen.

\section{AMD Staging}

Age-related macular degeneration staging was performed by grading of retinal images according to the standard protocol of the Cologne Image Reading Center (CIRCL) by certified graders. Age-related macular degeneration was classified by the presence of pigmentary changes together with at least 10 small drusen $(<63 \mu \mathrm{m})$ or presence of intermediate (63-124 $\mu \mathrm{m}$, early AMD) or the presence of large drusen $(\geq 125 \mu \mathrm{m}$ diameter) or $\geq 15$ intermediate drusen or geographic atrophy secondary to AMD outside the foveal central subfield (FCS, intermediate AMD) in the Early Treatment Diabetic Retinopathy Study (ETDRS) grid on FP. The subgroup of late AMD was defined as either AMD with geographic atrophy inside the FCS and/or CNV in at least one eye. Geographic atrophy was defined as sharply demarcated round or oval areas of depigmentation of the RPE of $\geq 175 \mu \mathrm{m}$ diameter with increased visibility of choroidal vessels on FP without signs of CNV. Late AMD with CNV was defined as CNV lesion within the ETDRS grid secondary to AMD either on FP, FA, or SD-OCT, when there was evidence for fluid, blood, or fibrovascular tissue on $\mathrm{FP}$, active classic or occult $\mathrm{CNV}$, or signs for previous $\mathrm{CNV}$, such as staining scar on FA and/or subretinal hyperreflective material, or fibrovascular pigment epithelial detachment (PED) on SD-OCT secondary to AMD.

Control subjects had to have no drusen, or only small drusen or pigmentary changes without or with less than 10 small drusen.

\section{Genotyping}

Genotyping of single nucleotide polymorphisms (SNPs) in the CFH (Y402H; rs1061170) and ARMS2 (A69S; rs10490924) gene was performed with a pre-designed TaqMan SNP genotyping assay for ARMS2 (Assay ID C_29934973_20, Applied Biosystems, Foster City, CA) and a custom TaqMan SNP genotyping assay for $\mathrm{CFH}$ (Applied Biosystems). The assays were analyzed on the ABI 7900HT system (Applied Biosystems) according to the protocols provided by the manufacturer.

\section{Statistical Analysis}

All calculations were carried out using SPSS software version 21.0 (IBM Software and Systems, Armonk, NY). Genetic associations of $C F H$ and ARMS2 with AMD risk were assessed by logistic regression analysis. Genotypes were coded as the number of AMD risk alleles (0, 1, and 2). For the logistic regression analyses of environmental factors, we included smoking (ever/never smoker), hypertension, diabetes, BMI (normal/overweight/obese), and sex. Odds ratios (OR) and 95\% confidence intervals (CI) were calculated for genetic risk alleles and environmental risk factors in unadjusted and adjusted models. Based on the stepwise logistic regression for genetic and environmental factors, risk scores were calculated with three logistic regression equations:

$$
\begin{aligned}
\operatorname{logit}(p 1)= & \log (p 1 /[1-p 1])=\beta 0+\beta 1^{*} \text { ARMS } 2 \\
& +\beta 2^{*} C F H
\end{aligned}
$$

where $p 1=$ risk for AMD under genetic influence;

$$
\begin{aligned}
\operatorname{logit}(p 2)= & \log (p 2 /[1-p 1])=\beta 0+\beta 1 * \text { smoking } \\
& +\beta 2 * \text { hypertension }+\beta 3^{*} B M I+\beta 4^{*} \text { diabetes } \\
& +\beta 5^{*} \text { sex }
\end{aligned}
$$

where $p 2=$ risk for AMD under environmental influence; and

$$
\begin{aligned}
\operatorname{logit}(p 3)= & \log (\not 3 /[1-p 3])=\beta 0+\beta 1^{*} \text { ARMS } 2+\beta 2^{*} C F H \\
& +\beta 1^{*} \text { smoking }+\beta 2^{*} \text { bypertension } \\
& +\beta 3^{*} B M I+\beta 4^{*} \text { diabetes }+\beta 5^{*} \text { sex }
\end{aligned}
$$

where $p 3=$ risk for $\mathrm{AMD}$ under genetic and environmental influence.

An estimate for the probability of AMD for each risk score was calculated with the equation $P=\exp (\operatorname{logit}[P]) /$ $(1+\exp [\operatorname{logit}\{P\}])$ and used to determine the receiver-operating-characteristics (ROC) curve.

\section{Results}

\section{Demographics}

This study included 2737 persons. The nonagenarian group included 166 persons with at least 90 years of age $(92.76 \pm$ 2.46 years; range, 90-100 years). Characteristics of all age groups are summarized in Table 1 .

\section{Associations of ARMS2 and CFH With AMD in Different Age Groups}

Associations with AMD were determined for the SNPs rs10490924 in ARMS2 and rs1061170 in $C F H$ for different age groups using logistic regression analysis (Table 2).

The weakest association for ARMS 2 was observed in the group of nonagenarians. Similarly, the association of $\mathrm{CFH}$ with AMD (and late AMD) increased continuously from the youngest group to the group of " $80-89$ years" and dropped down for persons aged more than 90 years.

An additional analysis adjusting for sex, site, smoking, hypertension, BMI, and diabetes yielded similar results (Table 3).

\section{Different Discriminative Ability of Computed Risk Scores for Different Age Groups}

Based on genetic risk alleles and environmental factors, three multiple logistic regression models were generated. In the first 
TABLE 1. Demographics

\begin{tabular}{|c|c|c|c|c|c|c|c|c|}
\hline & \multicolumn{2}{|c|}{$<70 \mathrm{y}$} & \multicolumn{2}{|c|}{$70-79 y$} & \multicolumn{2}{|c|}{$80-89$ y } & \multicolumn{2}{|c|}{ Nonagenarians } \\
\hline & No AMD & AMD & No AMD & AMD & No AMD & AMD & No AMD & AMD \\
\hline$n$ & 669 & 315 & 496 & 603 & 87 & 401 & 52 & 114 \\
\hline \multicolumn{9}{|l|}{$\operatorname{Sex}(n / \%)$} \\
\hline Female & $398 / 40.5 \%$ & $189 / 60.0 \%$ & $274 / 55.2 \%$ & $357 / 59.2 \%$ & $46 / 52.9 \%$ & $257 / 64.1 \%$ & $35 / 67.3 \%$ & $76 / 66.7 \%$ \\
\hline Male & $271 / 59.5 \%$ & $126 / 40.0 \%$ & $222 / 44.8 \%$ & $246 / 40.8 \%$ & $41 / 47.1 \%$ & $144 / 35.9 \%$ & $17 / 32.7 \%$ & $38 / 33.3 \%$ \\
\hline $\begin{array}{l}\text { Mean age } \pm \text { SD } \\
\text { Site }(n / \%)\end{array}$ & $64.68 \pm 4.13$ & $65.06 \pm 3.77$ & $73.48 \pm 2.76$ & $74.72 \pm 2.87$ & $82.58 \pm 2.42$ & $83.25 \pm 2.61$ & $92.96 \pm 2.26$ & $92.66 \pm 2.55$ \\
\hline UK & $335 / 50.1 \%$ & $135 / 42.9 \%$ & $235 / 47.4 \%$ & $284 / 47.1 \%$ & $38 / 43.7 \%$ & $191 / 47.6 \%$ & $49 / 94.2 \%$ & $98 / 86.0 \%$ \\
\hline $\mathrm{UMCN}$ & $334 / 49.9 \%$ & $180 / 57.1 \%$ & $261 / 52.6 \%$ & $319 / 52.9 \%$ & $49 / 56.3 \%$ & $210 / 52.4 \%$ & $3 / 5.8 \%$ & $16 / 14.0 \%$ \\
\hline Smoking & $385 / 59.3 \%$ & $177 / 56.2 \%$ & $271 / 54.6 \%$ & $321 / 60.9 \%$ & $41 / 50.0 \%$ & $158 / 50.0 \%$ & $24 / 46.2 \%$ & $43 / 42.2 \%$ \\
\hline Hypertension & $231 / 35.1 \%$ & $97 / 33.1 \%$ & $211 / 43.1 \%$ & $203 / 35.7 \%$ & $38 / 44.7 \%$ & $104 / 29.0 \%$ & $28 / 54.9 \%$ & $46 / 40.4 \%$ \\
\hline Diabetes & $45 / 6.8 \%$ & $22 / 7.5 \%$ & $42 / 8.6 \%$ & $67 / 11.8 \%$ & $7 / 8.2 \%$ & $33 / 9.2 \%$ & $4 / 7.8 \%$ & $14 / 12.8 \%$ \\
\hline \multicolumn{9}{|l|}{ BMI } \\
\hline$<25$ & $276 / 44.0 \%$ & $99 / 36.8 \%$ & $158 / 34.4 \%$ & $189 / 36.1 \%$ & $29 / 37.2 \%$ & $130 / 40.6 \%$ & $23 / 53.5 \%$ & $57 / 59.4 \%$ \\
\hline $25-29.9$ & $258 / 41.1 \%$ & $121 / 45.0 \%$ & $235 / 51.2 \%$ & $256 / 48.9 \%$ & $40 / 51.3 \%$ & $150 / 46.9 \%$ & $17 / 39.5 \%$ & $34 / 35.4 \%$ \\
\hline$\geq 30$ & $93 / 14.8 \%$ & $49 / 18.2 \%$ & $66 / 14.4 \%$ & $78 / 14.9 \%$ & $9 / 11.5 \%$ & $40 / 12.5 \%$ & $3 / 7.0 \%$ & $5 / 5.2 \%$ \\
\hline
\end{tabular}

UK, University Hospital of Cologne, Germany; UMCN, The Radboud University Nijmegen, the Netherlands.

model risk alleles of the two SNPs in $C F H$ and ARMS2 were used as predictive variables to compute genetic risk scores for each individual (model 1). An environmental risk score was calculated in a similar fashion (model 2). A general risk score was generated from all genetic and environmental factors (model 3, Table 4).

The highest classification efficiency in model 1 was observed in the group " $80-89$ years" (area-under-curve $[\mathrm{AUC}]=0.768$ for $\mathrm{AMD}$ versus No AMD, $\mathrm{AUC}=0.797$ for late AMD versus No AMD) and diminished in the nonagenarian group $(\mathrm{AUC}=0.659$ for $\mathrm{AMD}$ versus No $\mathrm{AMD}, \mathrm{AUC}=0.717$ for late AMD versus No AMD). Analysis of only environmental risk score (model 2) showed poor classification efficiency for all age groups and the combined model 3 revealed similar results to model 1 with only marginally better classification.

\section{Differences of Genetic Associations Within Different Age Groups}

The Figure presents the minor allele frequencies (MAFs) in different age groups.

For both SNPs, there was a decrease of MAFs with increasing age in controls and AMD subgroups (AMD and late AMD), especially visible in the comparison of "80-89 years" with nonagenarians. The only exceptions for this pattern were the MAFs of ARMS 2 in controls: the MAFs were highest in the

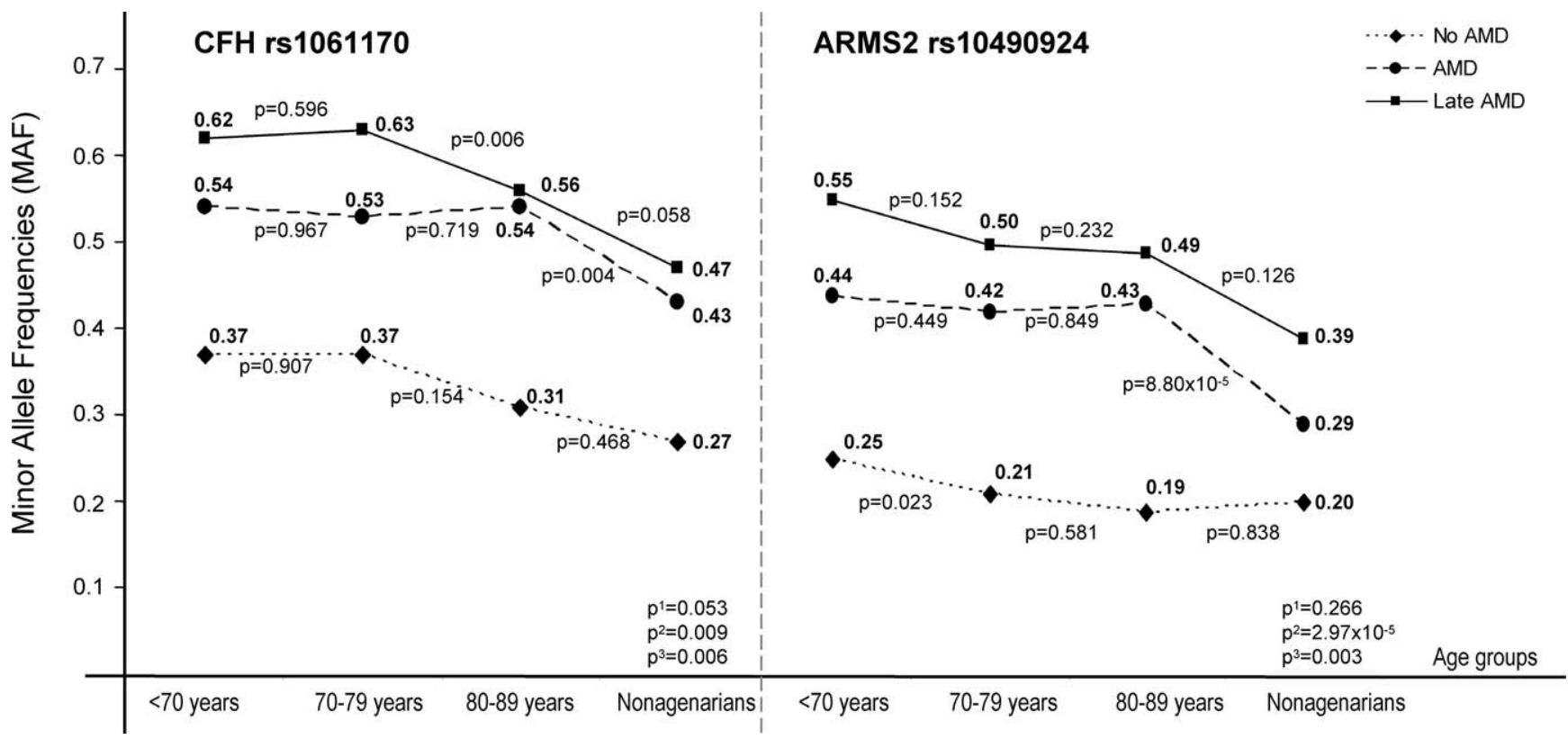

FiguRE. Minor allele frequencies of $C F H$ and $A R M S 2$ in different age groups and different AMD stages. Minor allele frequencies are compared using $\chi^{2}$ test. $P$ values of the $\chi^{2}$ test comparing " $<70$ years" with "nonagenarians" are presented as $P^{1}$ for no AMD cases, $P^{2}$ for AMD cases, and $P^{3}$ for late AMD cases. 
TABLE 2. Associations of $A R M S 2$ and $C F H$ With AMD in Different Age Groups

\begin{tabular}{|c|c|c|c|c|c|c|}
\hline & \multicolumn{3}{|c|}{ AMD vs. No AMD } & \multicolumn{3}{|c|}{ Late AMD vs. No AMD } \\
\hline & OR & $P$ Value & $95 \% \mathrm{CI}$ & OR & $P$ Value & $95 \% \mathrm{CI}$ \\
\hline \multicolumn{7}{|l|}{ ARMS2 } \\
\hline$<70 \mathrm{y}$ & 2.23 & $1.03 \times 10^{-13}$ & $1.83-2.73$ & 3.34 & $1.00 \times 10^{-13}$ & $2.55-4.40$ \\
\hline $70-79 y$ & 2.70 & $1.00 \times 10^{-13}$ & $2.22-3.29$ & 3.58 & $1.00 \times 10^{-13}$ & $2.84-4.50$ \\
\hline $80-89$ y & 3.11 & $6.56 \times 10^{-8}$ & $2.06-4.71$ & 3.63 & $2.75 \times 10^{-9}$ & $2.37-5.55$ \\
\hline Nonagenarians & 1.52 & 0.127 & $0.89-2.62$ & 2.40 & 0.004 & $1.31-4.37$ \\
\hline \multicolumn{7}{|l|}{$\mathrm{CFH}$} \\
\hline$<70 \mathrm{y}$ & 1.96 & $1.80 \times 10^{-11}$ & $1.61-2.38$ & 2.76 & $6.68 \times 10^{-13}$ & $2.10-3.64$ \\
\hline $70-79 y$ & 1.89 & $4.48 \times 10^{-13}$ & $1.59-2.24$ & 2.87 & $1.00 \times 10^{-13}$ & $2.33-3.56$ \\
\hline $80-89 y$ & 2.71 & $1.28 \times 10^{-7}$ & $1.87-3.93$ & 2.97 & $2.87 \times 10^{-8}$ & $2.02-4.36$ \\
\hline Nonagenarians & 2.21 & 0.005 & $1.28-3.82$ & 2.34 & 0.004 & $1.31-4.17$ \\
\hline
\end{tabular}

TABLE 3. Associations of $A R M S 2$ and $C F H$ With AMD in Different Age Groups in an Adjusted Model

\begin{tabular}{|c|c|c|c|c|c|c|}
\hline & \multicolumn{3}{|c|}{ AMD vs. No AMD Adjusted* } & \multicolumn{3}{|c|}{ Late vs. No AMD Adjusted* } \\
\hline & OR & $P$ Value & 95\% CI & OR & $P$ Value & $95 \% \mathrm{CI}$ \\
\hline \multicolumn{7}{|l|}{ ARMS2 } \\
\hline$<70 \mathrm{y}$ & 2.14 & $7.39 \times 10^{-12}$ & $1.720-2.655$ & 3.77 & $1.01 \times 10^{-13}$ & $2.738-5.195$ \\
\hline $70-79 y$ & 2.73 & $1.00 \times 10^{-13}$ & $2.197-3.393$ & 3.67 & $1.00 \times 10^{-14}$ & $2.835-4.759$ \\
\hline $80-89$ y & 2.85 & $2.67 \times 10^{-6}$ & $1.839-4.403$ & 3.36 & $2.12 \times 10^{-7}$ & $2.125-5.310$ \\
\hline Nonagenarians & 1.39 & 0.293 & $0.754-2.541$ & 2.35 & 0.018 & $1.160-4.749$ \\
\hline \multicolumn{7}{|l|}{$\mathrm{CFH}$} \\
\hline$<70 \mathrm{y}$ & 1.83 & $1.95 \times 10^{-8}$ & $1.481-2.257$ & 2.63 & $6.99 \times 10^{-10}$ & $1.932-3.567$ \\
\hline $70-79 y$ & 1.90 & $9.74 \times 10^{-12}$ & $1.578-2.281$ & 3.22 & $1.00 \times 10^{-13}$ & $2.529-4.094$ \\
\hline $80-89 y$ & 2.92 & $5.17 \times 10^{-7}$ & $1.920-4.430$ & 3.51 & $5.28 \times 10^{-8}$ & $2.233-5.517$ \\
\hline Nonagenarians & 2.34 & 0.008 & $1.250-4.368$ & 2.38 & 0.013 & $1.197-4.742$ \\
\hline
\end{tabular}

* Adjusted model with covariates smoking, hypertension, diabetes, body mass index, site, and sex.

youngest group (0.25), decreasing to 0.19 in the group of " 80 89 " years and in the nonagenarian group $(0.20)$

The OR for the nonagenarian group (nonagenarian versus nonnonagenarian) was estimated 0.74 for $C F H(P=0.085 ; 95 \%$ CI, 0.53-1.04) and 0.61 for ARMS2 ( $P=0.007$; 95\% CI, $0.43-$ 0.87) using a logistic regression model adjusting for AMD

\section{Discussion}

In this study we analyzed the age-dependent association of genetic and environmental risk factors for AMD, and compared very old persons aged $90-100$ years with different age groups. While the associations for the two major genetic risk factors ARMS2 (rs10490924) and CFH (rs1061170) were strong in persons aged less than 90 years with continuously rising OR

TABLE 4. Discrimination Accuracy of Genetic and Environmental Risk Scores

RS, risk score.

* Genetic RS based on multivariate logistic regression, including ARMS2 and CFH. pattern from the youngest group to the group of "80-89 years," this association was much weaker for the nonagenarian group. We also found significantly reduced risk allele frequencies in nonagenarians compared to the youngest group for the AMD phenotype, although the risk allele frequencies in controls remained relatively stable without significant difference. These findings were supported by risk score calculations using logistic regression analysis, demonstrating that $\mathrm{CFH}$ and ARMS2 risks alleles have a weaker role in AMD at very advanced age. In addition, no difference in environmental factors was observed between nonagenarians and younger AMD patients. This suggested that other genetic and environmental factors may be involved in the development of AMD in this age group. In addition, one can speculate that risk alleles in CFH and ARMS2 are associated with increased mortality.

\begin{tabular}{|c|c|c|c|c|c|c|}
\hline & \multicolumn{3}{|c|}{ AUC for Discrimination AMD vs. No AMD } & \multicolumn{3}{|c|}{ AUC for Discrimination Late AMD vs. No AMD } \\
\hline & Genetic RS* & Environmental RS $\dagger$ & General RS $\ddagger$ & Genetik RS & Environmental RS & General RS \\
\hline$<70 \mathrm{y}$ & 0.682 & 0.579 & 0.689 & 0.768 & 0.608 & 0.801 \\
\hline $70-79 y$ & 0.704 & 0.567 & 0.714 & 0.784 & 0.603 & 0.809 \\
\hline $80-89 y$ & 0.768 & 0.600 & 0.787 & 0.797 & 0.618 & 0.825 \\
\hline Nonagenarians & 0.659 & 0.608 & 0.682 & 0.717 & 0.666 & 0.742 \\
\hline
\end{tabular}

† Environmental RS based on multiple logistic regression, including hypertension, diabetes, body mass index, smoking, diabetes, site, sex

‡ General RS based on multivariate logistic regression, including environmental and genetic factors. 
A similar age-dependent association of $\mathrm{CFH}$ was described previously by Adams et al. ${ }^{17}$ where the prevalence of AMD in persons homozygous for the $\mathrm{CFH}$ risk variant was decreased in older persons (age range from $48-86$ years). Grassmann et al. ${ }^{18}$ also reported relatively lower associations of 13 AMD risk variants with AMD in an elderly group ( $>75$ years) in comparison with a younger group ( $<75$ years). The phenomenon of genetic differences between younger and older populations is widely described in longevity studies and explained as a result of differential survival, with an enrichment of "longevity genes" in the elderly. ${ }^{19-21}$ Lower effect sizes (ORs) of genetic risk alleles in ARMS2 and $C F H$ on the development of AMD, and lower risk allele frequencies in nonagenarian AMD patients may be caused by increased mortality of AMD patients carrying these alleles. Differential survival by AMD has been investigated in other studies. Some found an increased mortality risk in persons with AMD, ${ }^{7,22,23}$ while others did not find this association. ${ }^{24-26}$ The AREDS Report No. 13 showed an association of AMD with increased mortality even after adjustment for potentially important covariates. $^{7}$ In contrast, in the Rotterdam Study, shorter survival of AMD patients was explained by systemic risk factors also affecting mortality: There was no significant association of AMD with mortality after adjustment for various systemic factors. ${ }^{24}$ The $C F H$ risk variant could be associated with an increased mortality ${ }^{27}$ by its reduced capacity to downregulate complement activation and control inflammation. ${ }^{28}$ In a longitudinal study of nonagenarians, increased mortality was observed among the carriers of the $C F H$ rs1061170 allele independent of comorbidities. ${ }^{27}$

The results presented here are based on a case-control study, and, thus, do not allow the analysis of longitudinal or epidemiologic parameters. Our study included a large nonagenarian group, who primarily came from a small area in Germany, which may increase the chance of a selection bias, especially as a bias toward more healthy and mobile nonagenarians is possible. Furthermore, our analysis was limited to two genetic polymorphisms and few environmental factors. An extended analysis including other genetic and environmental factors may identify effects that explain AMD in the nonagenarian population. It must be noted that environmental factors may change over time as well. Therefore, the nonagenarian group cannot be matched easily with younger populations. For example, it is unknown what time span in life influences AMD development. The allele frequencies and effect sizes of ARMS2 and $C F H$ SNPs in the younger group were comparable to those in other studies. ${ }^{18,23,29}$

In summary, in our study genetic risk alleles in $\mathrm{CFH}$ and ARMS2 showed significantly smaller effect on AMD development in nonagenarians, while environmental factors retained a similar effect in advanced age. Larger epidemiologic studies with more statistical power are needed to investigate the role of $C F H$ and ARMS2 in nonagenarians and to validate our results. The verification of the enrichment of nonrisk allele frequencies of $C F H$ and $A R M S 2$ in a long-lived population may indicate a genetic influence of $C F H$ and $A R M S 2$ on mortality.

\section{Acknowledgments}

Disclosure: L. Ersoy, None; T. Ristau, None; M. Hahn, None; M. Karlstetter, None; T. Langmann, None; K. Dröge, None; A. Caramoy, None; A.I. den Hollander, None; S. Fauser, None

\section{References}

1. Kahn HA, Leibowitz HM, Ganley JP, et al. The Framingham Eye Study. II. Association of ophthalmic pathology with single variables previously measured in the Framingham Heart Study. Am J Epidemiol. 1977;106:33-41.
2. Sperduto RD, Hiller R. Systemic hypertension and age-related maculopathy in the Framingham Study. Arch Ophthalmol. 1986;104:216-219.

3. Klein R, Klein BE, Moss SE. Diabetes, hyperglycemia, and agerelated maculopathy. The Beaver Dam Eye Study. Ophthalmology. 1992;99:1527-1534.

4. Klein R, Klein BE, Tomany SC, Cruickshanks KJ. The association of cardiovascular disease with the long-term incidence of age-related maculopathy: the Beaver Dam Eye Study. Ophthalmology. 2003;110:1273-1280.

5. Hyman L, Schachat AP, He Q, Leske MC. Hypertension, cardiovascular disease, and age-related macular degeneration. Age-Related Macular Degeneration Risk Factors Study Group. Arch Ophthalmol. 2000;118:351-358.

6. Seddon JM, Cote J, Davis N, Rosner B. Progression of agerelated macular degeneration: association with body mass index, waist circumference, and waist-hip ratio. Arch $O p b$ thalmol. 2003;121:785-792.

7. Clemons TE, Kurinij N, Sperduto RD. Associations of mortality with ocular disorders and an intervention of high-dose antioxidants and zinc in the Age-Related Eye Disease Study: AREDS Report No. 13. Arch Ophthalmol. 2004;122:716-726.

8. Cougnard-Gregoire A, Delyfer MN, Korobelnik JF, et al. Longterm blood pressure and age-related macular degeneration: The ALIENOR Study. Invest Ophthalmol Vis Sci. 2013;54: 1905-1912.

9. Seddon JM, Reynolds R, Maller J, et al. Prediction model for prevalence and incidence of advanced age-related macular degeneration based on genetic, demographic, and environmental variables. Invest Ophthalmol Vis Sci. 2009;50:20442053.

10. Spencer KL, Olson LM, Schnetz-Boutaud N, et al. Using genetic variation and environmental risk factor data to identify individuals at high risk for age-related macular degeneration. PLoS One. 2011;6:e17784.

11. Weger M, Renner W, Steinbrugger I, et al. Association of the HTRA $1-625 \mathrm{G}>\mathrm{A}$ promoter gene polymorphism with exudative age-related macular degeneration in a Central European population. Mol Vis. 2007;13:1274-1279.

12. Hageman GS, Anderson DH, Johnson LV, et al. A common haplotype in the complement regulatory gene factor $\mathrm{H}$ (HF1/ $\mathrm{CFH})$ predisposes individuals to age-related macular degeneration. Proc Natl Acad Sci U S A. 2005;102:7227-7232.

13. Rivera A, Fisher SA, Fritsche LG, et al. Hypothetical LOC387715 is a second major susceptibility gene for agerelated macular degeneration, contributing independently of complement factor $\mathrm{H}$ to disease risk. Hum Mol Genet. 2005; 14:3227-3236.

14. Dewan A, Liu M, Hartman S, et al. HTRA1 promoter polymorphism in wet age-related macular degeneration. Science. 2006;314:989-992.

15. Vierkotten S, Muether PS, Fauser S. Overexpression of HTRA1 leads to ultrastructural changes in the elastic layer of Bruch's membrane via cleavage of extracellular matrix components. PLoS One. 2011;6:e22959.

16. Hermann M, Caramoy A, Schroder S, et al. Prevalence of agerelated macular degeneration in persons aged 90 years and older in Cologne. Acta Ophthalmol. 2012;90:e500-501.

17. Adams MK, Simpson JA, Richardson AJ, et al. Can genetic associations change with age? $\mathrm{CFH}$ and age-related macular degeneration. Hum Mol Genet. 2012;21:5229-5236.

18. Grassmann F, Fritsche LG, Keilhauer CN, Heid IM, Weber BH. Modelling the genetic risk in age-related macular degeneration. PLoS One. 2012;7:e37979.

19. Slagboom PE, Beekman M, Passtoors WM, et al. Genomics of human longevity. Philos Trans $R$ Soc Lond B Biol Sci. 2011; 366:35-42. 
20. Kenyon CJ. The genetics of ageing. Nature. 2010;464:504512.

21. Beekman M, Blanche H, Perola M, et al. Genome-wide linkage analysis for human longevity: Genetics of Healthy Aging Study. Aging Cell. 2013;12:184-193.

22. Buch H, Vinding T, la Cour M, et al. Age-related maculopathy: a risk indicator for poorer survival in women: the Copenhagen City Eye Study. Ophthalmology. 2005;112:305-312.

23. Gangnon RE, Lee KE, Klein BE, et al. Effect of the $\mathrm{Y} 402 \mathrm{H}$ variant in the complement factor $\mathrm{H}$ gene on the incidence and progression of age-related macular degeneration: results from multistate models applied to the Beaver Dam Eye Study. Arch Ophthalmol. 2012;130:1169-1176.

24. Borger PH, van Leeuwen R, Hulsman CA, et al. Is there a direct association between age-related eye diseases and mortality? The Rotterdam Study. Ophthalmology. 2003;110:1292-1296.
25. Tan JS, Wang JJ, Liew G, Rochtchina E, Mitchell P. Age-related macular degeneration and mortality from cardiovascular disease or stroke. Br J Ophthalmol. 2008;92:509-512.

26. $\mathrm{Xu} \mathrm{L,} \mathrm{Li} \mathrm{YB,} \mathrm{Wang} \mathrm{YX,} \mathrm{Jonas} \mathrm{JB.} \mathrm{Age-related} \mathrm{macular}$ degeneration and mortality: the Beijing eye study. Ophthalmologica. 2008;222:378-379.

27. Jylhava J, Eklund C, Jylha M, et al. Complement factor $H$ 402His variant confers an increased mortality risk in Finnish nonagenarians: the Vitality $90+$ study. Exp Gerontol. 2009;44: 297-299.

28. Laine M, Jarva H, Seitsonen S, et al. Y402H polymorphism of complement factor $\mathrm{H}$ affects binding affinity to C-reactive protein. J Immunol. 2007;178:3831-3836.

29. Chakravarthy U, McKay GJ, de Jong PT, et al. ARMS2 increases the risk of early and late age-related macular degeneration in the European Eye Study. Ophthalmology. 2013;120:342-348. 